\title{
Common Fixed Point Theorems of Meir and Keeler Type for Weakly Compatible Maps
}

\author{
H. Bouhadjera AND Brian Fisher
}

\begin{abstract}
The subject of this paper is to prove a common fixed point theorem for four weakly compatible maps which extends and improves Theorem 1 of [2] and others by removing the assumption of continuity and relaxing the property of compatibility to weak compatibility. Also we give another extension of the same theorem.
\end{abstract}

\section{INTRODUCTION AND PRELIMINARIES}

In 1986, Jungck [3] introduced generalized commuting maps, called compatible maps, which are more general than the concept of weakly commuting maps. Let $\mathcal{S}$ and $\mathcal{T}$ be two self maps of a metric space $(\mathcal{X}, d)$. He defines $\mathcal{S}$ and $\mathcal{T}$ to be compatible if

$$
\lim _{n \rightarrow \infty} d\left(\mathcal{S} \mathcal{T} x_{n}, \mathcal{T} \mathcal{S} x_{n}\right)=0
$$

whenever $\left\{x_{n}\right\}$ is a sequence in $\mathcal{X}$ such that $\lim _{n \rightarrow \infty} \mathcal{S} x_{n}=\lim _{n \rightarrow \infty} \mathcal{T} x_{n}=t$ for some $t \in \mathcal{X}$. This concept has been useful as a tool for obtaining more comprehensive fixed point theorems. In general, commuting maps are weakly commuting and weakly commuting maps are compatible, but the converses are not necessarily true $([3])$.

In 1993, G. Jungck, P.P. Murthy and Y.J. Cho [6] introduced the concept of compatible mappings of type $(A)$ as follows: $\mathcal{S}$ and $\mathcal{T}$ above are compatible of type $(A)$ if in lieu of $(1)$, we have

$$
\lim _{n \rightarrow \infty} d\left(\mathcal{S} \mathcal{T} x_{n}, \mathcal{T}^{2} x_{n}\right)=0 \quad \text { and } \quad \lim _{n \rightarrow \infty} d\left(\mathcal{T} \mathcal{S} x_{n}, \mathcal{S}^{2} x_{n}\right)=0
$$

The notions of compatible and compatible maps of type $(A)$ are independent (see $[6])$.

In 1995, H. K. Pathak and M. S. Khan [8] gave a generalization of compatible maps of type $(A)$ by introducing the concept of compatible maps of type $(B)$.

2000 Mathematics Subject Classification. Primary: 7H10, 54H25.

Key words and phrases. Weakly compatible maps, compatible and compatible maps of type $(\mathrm{A}),(\mathrm{B}),(\mathrm{P})$ and $(\mathrm{C})$, common fixed point theorems. 
$\mathcal{S}$ and $\mathcal{T}$ above are said to be compatible of type $(B)$ if instead of (1) we have the two inequalities

$$
\lim _{n \rightarrow \infty} d\left(\mathcal{S} \mathcal{T} x_{n}, \mathcal{T}^{2} x_{n}\right) \leq \frac{1}{2}\left[\lim _{n \rightarrow \infty} d\left(\mathcal{S T} x_{n}, \mathcal{S} t\right)+\lim _{n \rightarrow \infty} d\left(\mathcal{S} t, \mathcal{S}^{2} x_{n}\right)\right]
$$

and

$$
\lim _{n \rightarrow \infty} d\left(\mathcal{T} \mathcal{S} x_{n}, \mathcal{S}^{2} x_{n}\right) \leq \frac{1}{2}\left[\lim _{n \rightarrow \infty} d\left(\mathcal{T} \mathcal{S} x_{n}, \mathcal{T} t\right)+\lim _{n \rightarrow \infty} d\left(\mathcal{T} t, \mathcal{T}^{2} x_{n}\right)\right]
$$

This definition is equivalent to the concept of compatible mappings of type $(A)$ under some conditions.

In [7] Pathak et al. introduced the compatibility of type $(P)$ and compared with the compatibility and the compatibility of type $(A)$. They define $\mathcal{S}$ and $\mathcal{T}$ above to be compatible of type $(P)$ if in place of $(1)$, we have

$$
\lim _{n \rightarrow \infty} d\left(\mathcal{S}^{2} x_{n}, \mathcal{T}^{2} x_{n}\right)=0
$$

In 1998, Pathak, Cho, Kang and Madharia [8] introduced a new extension of compatible maps of type $(A)$ by giving the notion of compatible maps of type $(C)$. They defined $\mathcal{S}$ and $\mathcal{T}$ above to be compatible of type $(C)$ if we have in lieu of (1) the two inequalities:

$$
\begin{gathered}
\lim _{n \rightarrow \infty} d\left(\mathcal{S T} x_{n}, \mathcal{T}^{2} x_{n}\right) \leq \frac{1}{3}\left[\lim _{n \rightarrow \infty} d\left(\mathcal{S T} x_{n}, \mathcal{S} t\right)+\lim _{n \rightarrow \infty} d\left(\mathcal{S} t, \mathcal{T}^{2} x_{n}\right)\right. \\
\left.+\lim _{n \rightarrow \infty} d\left(\mathcal{S} t, \mathcal{S}^{2} x_{n}\right)\right]
\end{gathered}
$$

and

$$
\begin{gathered}
\lim _{n \rightarrow \infty} d\left(\mathcal{T S} x_{n}, \mathcal{S}^{2} x_{n}\right) \leq \frac{1}{3}\left[\lim _{n \rightarrow \infty} d\left(\mathcal{T} \mathcal{S} x_{n}, \mathcal{T} t\right)+\lim _{n \rightarrow \infty} d\left(\mathcal{T} t, \mathcal{S}^{2} x_{n}\right)\right. \\
\left.+\lim _{n \rightarrow \infty} d\left(\mathcal{T} t, \mathcal{T}^{2} x_{n}\right)\right]
\end{gathered}
$$

As is obvious from the definitions, compatible maps of type $(B)$ and $(C)$ generalize those of compatible maps of type $(A)$.

Recently, Jungck gave in his paper [4] a new generalization of the compatibility by introducing the concept of weakly compatible mappings. He defines $\mathcal{S}$ and $\mathcal{T}$ above to be weakly compatible if $\mathcal{S} t=\mathcal{T} t, t \in \mathcal{X}$ implies $\mathcal{S} \mathcal{T} t=\mathcal{T} \mathcal{S} t$.

It is clear that every compatible (compatible of type $(A),(B),(P),(C)$ ) pair of mappings is weakly compatible. The following example is an example of weakly compatible maps which are not compatible (compatible of type $(A),(B),(P)$, $(C))$. 
Example 1.1. Let $\mathcal{X}=[0, \infty)$ be with the absolute value metric. Define mappings $\mathcal{S}, \mathcal{T}: \mathcal{X} \rightarrow \mathcal{X}$ by

$$
\mathcal{S} x=\left\{\begin{array}{ll}
x & \text { if } x \in[0,1), \\
1 & \text { if } x=1, \\
3 & \text { if } x \in(1, \infty),
\end{array} \quad \mathcal{T} x= \begin{cases}2-x & \text { if } x \in[0,1), \\
1 & \text { if } x=1, \\
6 & \text { if } x \in(1, \infty) .\end{cases}\right.
$$

$\mathcal{S}$ and $\mathcal{T}$ are weakly compatible since they commute at their coincidence point $t=1$. We assert that $\mathcal{S}$ and $\mathcal{T}$ are neither compatible nor compatible of type $(A)$, $(B),(P)$ and $(C)$. For that purpose let us suppose that $\left\{x_{n}\right\}$ is a sequence in $\mathcal{X}$ such that $x_{n}=1-\frac{1}{n}$ for $n \in \mathbb{N}^{*}$. We have

$$
\begin{array}{rlrl}
\mathcal{S} x_{n} & =x_{n} \rightarrow 1 ; & \mathcal{T} x_{n} & =2-x_{n} \rightarrow 1 \text { as } n \rightarrow \infty, \\
\mathcal{S} \mathcal{T} x_{n} & =\mathcal{S}\left(2-x_{n}\right)=3 ; & \mathcal{T} \mathcal{S} x_{n} & =\mathcal{T}\left(x_{n}\right)=2-x_{n}, \\
\mathcal{S S} x_{n} & =\mathcal{S}\left(x_{n}\right)=x_{n} ; & \mathcal{T} \mathcal{T} x_{n}=\mathcal{T}\left(2-x_{n}\right)=6 .
\end{array}
$$

Thus we have

$$
\lim _{n \rightarrow \infty} d\left(\mathcal{S} \mathcal{T} x_{n}, \mathcal{T} \mathcal{S} x_{n}\right)=\lim _{n \rightarrow \infty}\left|3-2+x_{n}\right|=2 \neq 0
$$

and so the pair $\{\mathcal{S}, \mathcal{T}\}$ is not compatible. Also, we have

$$
\lim _{n \rightarrow \infty} d\left(\mathcal{S} \mathcal{T} x_{n}, \mathcal{T}^{2} x_{n}\right)=\lim _{n \rightarrow \infty}|3-6|=3 \neq 0
$$

which implies that the pair $\{\mathcal{S}, \mathcal{T}\}$ is not compatible of type $(A)$. Further, we have

$$
\begin{aligned}
3 & =\lim _{n \rightarrow \infty} d\left(\mathcal{S T} x_{n}, \mathcal{T}^{2} x_{n}\right) \\
& \nless \leq \frac{1}{2}\left[\lim _{n \rightarrow \infty} d\left(\mathcal{S T} x_{n}, \mathcal{S} t\right)+\lim _{n \rightarrow \infty} d\left(\mathcal{S} t, \mathcal{S}^{2} x_{n}\right)\right] \\
& =\frac{1}{2}\left[\lim _{n \rightarrow \infty}|3-1|+\lim _{n \rightarrow \infty}\left|1-x_{n}\right|\right]=1,
\end{aligned}
$$

and so $\mathcal{S}$ and $\mathcal{T}$ are not compatible of type $(B)$.

Again, we have

$$
\lim _{n \rightarrow \infty} d\left(\mathcal{S}^{2} x_{n}, \mathcal{T}^{2} x_{n}\right)=\lim _{n \rightarrow \infty}\left|x_{n}-6\right|=5 \neq 0,
$$

which tells us that $\mathcal{S}$ and $\mathcal{T}$ are noncompatible of type $(P)$.

Finally, we have

$$
\begin{aligned}
3 & =\lim _{n \rightarrow \infty} d\left(\mathcal{S} \mathcal{T} x_{n}, \mathcal{T}^{2} x_{n}\right) \\
& \not \leq \frac{1}{3}\left[\lim _{n \rightarrow \infty} d\left(\mathcal{S T} x_{n}, \mathcal{S} t\right)+\lim _{n \rightarrow \infty} d\left(\mathcal{S} t, \mathcal{S}^{2} x_{n}\right)+\lim _{n \rightarrow \infty} d\left(\mathcal{S} t, \mathcal{T}^{2} x_{n}\right)\right] \\
& =\frac{1}{3}\left[\lim _{n \rightarrow \infty}|3-1|+\lim _{n \rightarrow \infty}\left|1-x_{n}\right|+\lim _{n \rightarrow \infty}|1-6|\right]=\frac{7}{3},
\end{aligned}
$$

and so the pair $\{\mathcal{S}, \mathcal{T}\}$ is noncompatible of type $(C)$.

For our main results we will need the following lemma given in [3]. 
Lemma 1.1. Let $(\mathcal{A}, \mathcal{S})$ and $(\mathcal{B}, \mathcal{T})$ be self mappings of a complete metric space $(\mathcal{X}, d)$ such that $\mathcal{A}(\mathcal{X}) \subset \mathcal{T}(\mathcal{X})$ and $\mathcal{B}(\mathcal{X}) \subset \mathcal{S}(\mathcal{X})$. Assume further that given $\epsilon>0$, there exists $\delta>0$ such that for all $x, y$ in $\mathcal{X}$

$$
\epsilon \leq M(x, y)<\epsilon+\delta \Rightarrow d(\mathcal{A} x, \mathcal{B} y)<\epsilon
$$

and

where

$$
d(\mathcal{A} x, \mathcal{B} y)<M(x, y), \text { whenever } M(x, y)>0
$$

$$
M(x, y)=\max \{d(\mathcal{S} x, \mathcal{T} y), d(\mathcal{A} x, \mathcal{S} x), d(\mathcal{B} y, \mathcal{T} y),[d(\mathcal{S} x, \mathcal{B} y)+d(\mathcal{A} x, \mathcal{T} y)]\}
$$

Then for each $x_{0}$ in $\mathcal{X}$, the sequence $\left\{y_{n}\right\}$ in $\mathcal{X}$ defined by the rule

$$
y_{2 n}=\mathcal{A} x_{2 n}=\mathcal{T} x_{2 n+1} \text { and } y_{2 n+1}=\mathcal{B} x_{2 n+1}=\mathcal{S} x_{2 n+2} \text { for } n \in \mathbb{N} .
$$

is a Cauchy sequence.

The following theorem was stated in [3].

Theorem 1.1. Let $(\mathcal{A}, \mathcal{S})$ and $(\mathcal{B}, \mathcal{T})$ be compatible pairs of self mappings of a complete metric space $(\mathcal{X}, d)$ such that

(i) $\mathcal{A}(\mathcal{X}) \subset \mathcal{T}(\mathcal{X})$ and $\mathcal{B}(\mathcal{X}) \subset \mathcal{S}(\mathcal{X})$

(ii) given $\epsilon>0$ there exists a $\delta>0$ such that for all $x, y$ in $\mathcal{X}$,

$$
\epsilon \leq M(x, y)<\epsilon+\delta \Rightarrow d(\mathcal{A} x, \mathcal{B} y)<\epsilon
$$

and

(iii) $d(\mathcal{A} x, \mathcal{B} y)<$

$<k[d(\mathcal{S} x, \mathcal{T} y)+d(\mathcal{A} x, \mathcal{S} x)+d(\mathcal{B} y, \mathcal{T} y)+d(\mathcal{S} x, \mathcal{B} y)+d(\mathcal{A} x, \mathcal{T} y)]$

for all $x, y$ in $\mathcal{X}$ where $0 \leq k<1 / 3$.

If one of the mappings $\mathcal{A}, \mathcal{B}, \mathcal{S}$ and $\mathcal{T}$ is continuous, then $\mathcal{A}, \mathcal{B}, \mathcal{S}$ and $\mathcal{T}$ have a unique common fixed point.

Note that Theorem 1.1 is not correct as it stands since if $\mathcal{A}, \mathcal{B}, \mathcal{S}$ and $\mathcal{T}$ had a fixed point $z$, then $(i i i)$ could not hold when $x=y=z$. The inequality $<$ in (iii) should be replaced by $\leq$.

\section{MAIN RESULtS}

Theorem 2.1. Let $(\mathcal{A}, \mathcal{S})$ and $(\mathcal{B}, \mathcal{T})$ be weakly compatible pairs of self mappings of a complete metric space $(\mathcal{X}, d)$ such that

(a) $\mathcal{A}(\mathcal{X}) \subset \mathcal{T}(\mathcal{X})$ and $\mathcal{B}(\mathcal{X}) \subset \mathcal{S}(\mathcal{X})$

(b) one of $\mathcal{A}(\mathcal{X}), \mathcal{B}(\mathcal{X}), \mathcal{S}(\mathcal{X})$ or $\mathcal{T}(\mathcal{X})$ is closed,

(c) given $\epsilon>0$ there exists a $\delta>0$ such that for all $x, y$ in $\mathcal{X}$,

$$
\epsilon \leq M(x, y)<\epsilon+\delta \Rightarrow d(\mathcal{A} x, \mathcal{B} y)<\epsilon
$$

where

$$
\begin{aligned}
& M(x, y)=\max \{d(\mathcal{S} x, \mathcal{T} y), d(\mathcal{A} x, \mathcal{S} x), d(\mathcal{B} y, \mathcal{T} y),[d(\mathcal{S} x, \mathcal{B} y)+d(\mathcal{A} x, \mathcal{T} y)] / 2\} \\
& \quad \text { and }
\end{aligned}
$$


(d) $d(\mathcal{A} x, \mathcal{B} y) \leq$

$$
\leq k \max \{d(\mathcal{S} x, \mathcal{T} y), d(\mathcal{A} x, \mathcal{S} x), d(\mathcal{B} y, \mathcal{T} y), d(\mathcal{S} x, \mathcal{B} y), d(\mathcal{A} x, \mathcal{T} y)\}
$$

for all $x, y$ in $X$ where $0 \leq k<1$.

Then, $\mathcal{A}, \mathcal{B}, \mathcal{S}$ and $\mathcal{T}$ have a unique common fixed point.

Proof. Let $x_{0}$ be an arbitrary point in $\mathcal{X}$, then, since $(a)$ holds, we can define inductively a sequence

$$
\left\{\mathcal{A} x_{0}, \mathcal{B} x_{1}, \mathcal{A} x_{2}, \mathcal{B} x_{3}, \ldots, \mathcal{A} x_{2 n}, \mathcal{B} x_{2 n+1}, \ldots\right\}
$$

such that

$$
y_{2 n}=\mathcal{A} x_{2 n}=\mathcal{T} x_{2 n+1} \text { and } y_{2 n+1}=\mathcal{B} x_{2 n+1}=\mathcal{S} x_{2 n+2} \text { for } n \in \mathbb{N} .
$$

By Lemma 1.1, it follows that $\left\{y_{n}\right\}$ is a Cauchy sequence in $\mathcal{X}$. Since $\mathcal{X}$ is complete, $\left\{y_{n}\right\}$ converges to some element $z \in \mathcal{X}$, as do the subsequences $\left\{\mathcal{A} x_{2 n}\right\}=$ $\left\{\mathcal{T} x_{2 n+1}\right\},\left\{\mathcal{S} x_{2 n}\right\}=\left\{\mathcal{B} x_{2 n-1}\right\}$ and $\left\{\mathcal{S} x_{2 n+2}\right\}=\left\{\mathcal{B} x_{2 n+1}\right\}$.

Suppose that $\mathcal{A}(\mathcal{X})$ is closed. Then since $\mathcal{A}(\mathcal{X}) \subset \mathcal{T}(\mathcal{X})$, there exists a point $u \in \mathcal{X}$ such that $z=\mathcal{T} u$. Using the inequality $(d)$, we have

$$
\begin{gathered}
d\left(\mathcal{A} x_{2 n}, \mathcal{B} u\right) \leq k \max \left\{d\left(\mathcal{S} x_{2 n}, \mathcal{T} u\right), d\left(\mathcal{A} x_{2 n}, \mathcal{S} x_{2 n}\right), d(\mathcal{B} u, \mathcal{T} u),\right. \\
\left.d\left(\mathcal{S} x_{2 n}, \mathcal{B} u\right), d\left(\mathcal{A} x_{2 n}, \mathcal{T} u\right)\right\} .
\end{gathered}
$$

Letting $n \rightarrow \infty$, we get

$$
d(z, \mathcal{B} u) \leq k d(z, \mathcal{B} u)
$$

which is a contradiction. Thus, $z=\mathcal{T} u=\mathcal{B} u$ and by the weak compatibility of $(\mathcal{B}, \mathcal{T})$, it follows that $\mathcal{B} \mathcal{T} u \mathcal{T} \mathcal{B} u$ and so $\mathcal{B} z=\mathcal{B} \mathcal{T} u=\mathcal{T} \mathcal{B} u=\mathcal{T} z$.

We claim that $z$ is a common fixed point of $\mathcal{B}$ and $\mathcal{T}$. Assume not. Then by inequality $(d)$, we obtain

$$
\begin{gathered}
d\left(\mathcal{A} x_{2 n}, \mathcal{B} z\right) \leq k \max \left\{d\left(\mathcal{S} x_{2 n}, \mathcal{T} z\right), d\left(\mathcal{A} x_{2 n}, \mathcal{S} x_{2 n}\right), d(\mathcal{B} z, \mathcal{T} z),\right. \\
\left.d\left(\mathcal{S} x_{2 n}, \mathcal{B} z\right), d\left(\mathcal{A} x_{2 n}, \mathcal{T} z\right)\right\} .
\end{gathered}
$$

Letting $n$ tends to infinity, it gives

$$
d(z, \mathcal{B} z) \leq k d(z, \mathcal{B} z)
$$

which implies that $z=\mathcal{B} z=\mathcal{T} z$.

Now, since $\mathcal{B}(\mathcal{X}) \subset \mathcal{S}(\mathcal{X})$, there exists a point $v \in \mathcal{X}$ such that $z=\mathcal{S} v$. Then, from inequality $(d)$, we have

$$
d(\mathcal{A} v, \mathcal{B} z) \leq k \max \{d(\mathcal{S} v, \mathcal{T} z), d(\mathcal{A} v, \mathcal{S} v), d(\mathcal{B} z, \mathcal{T} z), d(\mathcal{S} v, \mathcal{B} z), d(\mathcal{A} v, \mathcal{T} z)\}
$$

It follows that

$$
d(\mathcal{A} v, z) \leq k d(\mathcal{A} v, z)
$$

a contradiction, which implies that $\mathcal{A} v=z$. Also, since $\mathcal{A} v=\mathcal{S} v=z$ and by the weak compatibility of $\mathcal{A}$ and $\mathcal{S}$, it follows that $\mathcal{S} \mathcal{A} v=\mathcal{A} \mathcal{S} v$ and so $\mathcal{S} z=\mathcal{S} \mathcal{A} v=$ $\mathcal{A S} v=\mathcal{A} z$.

Again the use of inequality $(d)$ gives

$$
d(\mathcal{A} z, \mathcal{B} z) \leq k\{d(\mathcal{S} z, \mathcal{T} z), d(\mathcal{A} z, \mathcal{S} z), d(\mathcal{B} z, \mathcal{T} z), d(\mathcal{S} z, \mathcal{B} z), d(\mathcal{A} z, \mathcal{T} z)\}
$$


i.e.

$$
d(\mathcal{A} z, z) \leq k d(\mathcal{A} z, z)
$$

Consequently, we have $\mathcal{A} z=z=\mathcal{S} z$. Hence, $z$ is a common fixed point of $\mathcal{A}, \mathcal{B}$, $\mathcal{S}$ and $\mathcal{T}$.

Finally, we prove the uniqueness of $z$. Indeed, suppose that $w$ is a second distinct common fixed point of $\mathcal{A}, \mathcal{B}, \mathcal{S}$ and $\mathcal{T}$. Then again using inequality $(d)$, we get

$$
d(\mathcal{A} z, \mathcal{B} w) \leq k\{d(\mathcal{S} z, \mathcal{T} w), d(\mathcal{A} z, \mathcal{S} z), d(\mathcal{B} w, \mathcal{T} w), d(\mathcal{S} z, \mathcal{B} w), d(\mathcal{A} z, \mathcal{T} w)\}
$$

that is

$$
d(z, w) \leq k d(z, w)
$$

a contradiction, which implies that $w=z$.

Similarly, we can obtain this conclusion by supposing $\mathcal{B}(\mathcal{X})(\operatorname{resp} . \mathcal{S}(\mathcal{X}), \mathcal{T}(\mathcal{X}))$ is closed.

Now, we give our second main result which is another generalization of Theorem 1 of [2] and for this, we need the following:

Lemma 2.1 ([4] (resp. [6], [7], [9])). Let $\mathcal{S}$ and $\mathcal{T}$ be compatible and compatible of type $(A)$ (resp. $(B),(P))$ self mappings of a metric space $(\mathcal{X}, d)$. If $\mathcal{S} t=\mathcal{T} t$ for some $t \in \mathcal{X}$, then $\mathcal{S} \mathcal{T} t=\mathcal{T} \mathcal{S}$.

Proposition 2.1 ([1]). Let $\mathcal{S}$ and $\mathcal{T}$ be compatible mappings of type $(C)$ from a metric space $(\mathcal{X}, d)$ into itself. Suppose that $\lim _{n \rightarrow \infty} \mathcal{S} x_{n}=\lim _{n \rightarrow \infty} \mathcal{T} x_{n}=t$ for some $t \in \mathcal{X}$. Then we have the following:

(1) $\lim _{n \rightarrow \infty} \mathcal{T} \mathcal{T} x_{n}=\mathcal{S} t$ if $\mathcal{S}$ is continuous at $t$,

(2) $\lim _{n \rightarrow \infty} \mathcal{S S} x_{n}=\mathcal{T} t$ if $\mathcal{T}$ is continuous at $t$,

(3) $\mathcal{S} \mathcal{T} t=\mathcal{T} \mathcal{S} t$ and $\mathcal{S} t=\mathcal{T} t$ if $\mathcal{S}$ and $\mathcal{T}$ are continuous at $t$.

Theorem 2.2. Let $\mathcal{A}, \mathcal{B}, \mathcal{S}$ and $\mathcal{T}$ be mappings from a complete metric space $(\mathcal{X}, d)$ into itself satisfying conditions $(a),(b),(c)$ and $(d)$ of Theorem 2.1. Further, if the pairs $(\mathcal{A}, \mathcal{S})$ and $(\mathcal{B}, \mathcal{T})$ are compatible and compatible of type $(A)$ (resp. $(B),(P)$ and $(C)$ ), then the four mappings have a unique common fixed point $z \in \mathcal{X}$.

Proof. Define the sequence $\left\{y_{n}\right\}$ as in the proof of Theorem 2.1. Then $\left\{y_{n}\right\}$ is a Cauchy sequence in $\mathcal{X}$ and converges with its subsequences to $z \in \mathcal{X}$. Suppose that $\mathcal{B}(\mathcal{X})$ is closed. Then since $\mathcal{B}(\mathcal{X}) \subset \mathcal{S}(\mathcal{X})$, there exists an element $u \in \mathcal{X}$ such that $z=\mathcal{S} u$. Using $(d)$ we obtain

$$
\begin{gathered}
d\left(\mathcal{A} u, \mathcal{B} x_{2 n+1}\right) \leq k \max \left\{d\left(\mathcal{S} u, \mathcal{T} x_{2 n+1}\right), d(\mathcal{A} u, \mathcal{S} u), d\left(\mathcal{B} x_{2 n+1}, \mathcal{T} x_{2 n+1}\right),\right. \\
\left.d\left(\mathcal{S} u, \mathcal{B} x_{2 n+1}\right), d\left(\mathcal{A} u, \mathcal{T} x_{2 n+1}\right)\right\}
\end{gathered}
$$

By letting $n \rightarrow \infty$ in the above inequality, it gives

$$
d(\mathcal{A} u, z) \leq k d(\mathcal{A} u, z)
$$


which is a contradiction. Thus, $\mathcal{A} u=z=\mathcal{S} u$. But $\mathcal{A}$ and $\mathcal{S}$ are compatible of type $(A)$ (resp. $(B),(P)$ ), then by Lemma 2.1 it follows that $\mathcal{A} \mathcal{S} u=\mathcal{S} \mathcal{A} u$ and thus, $\mathcal{A} z=\mathcal{S} z$.

We claim that $z$ is a fixed point of $\mathcal{A}$ and $\mathcal{S}$. Suppose not, then by assumption $(d)$, we get

$$
\begin{gathered}
d\left(\mathcal{A} z, \mathcal{B} x_{2 n+1}\right) \leq k \max \left\{d\left(\mathcal{S} z, \mathcal{T} x_{2 n+1}\right), d(\mathcal{A} z, \mathcal{S} z), d\left(\mathcal{B} x_{2 n+1}, \mathcal{T} x_{2 n+1}\right),\right. \\
\left.d\left(\mathcal{S} z, \mathcal{B} x_{2 n+1}\right), d\left(\mathcal{A} z, \mathcal{T} x_{2 n+1}\right)\right\}
\end{gathered}
$$

Therefore as $n \rightarrow \infty$

$$
d(\mathcal{A} z, z) \leq k d(\mathcal{A} z, z)
$$

a contradiction which implies that $z=\mathcal{A} z=\mathcal{S} z$.

Now, since $\mathcal{A}(\mathcal{X}) \subset \mathcal{T}(\mathcal{X})$, then there exists a point $v \in \mathcal{X}$ such that $z=\mathcal{A} z=$ $\mathcal{T} v$. The use of inequality $(d)$ gives

$$
d(\mathcal{A} z, \mathcal{B} v) \leq k \max \{[d(\mathcal{S} z, \mathcal{T} v), d(\mathcal{A} z, \mathcal{S} z), d(\mathcal{B} v, \mathcal{T} v), d(\mathcal{S} z, \mathcal{B} v), d(\mathcal{A} z, \mathcal{T} v)\}
$$

It follows that

$$
d(z, \mathcal{B} v) \leq k d(z, \mathcal{B} v)
$$

which is a contradiction, so we have $\mathcal{B} v=z=\mathcal{T} v$. Since $\mathcal{B}$ and $\mathcal{T}$ are compatible, compatible of type $(A)$ (resp. $(B),(P))$, by Lemma 2.1 it follows that $\mathcal{B} \mathcal{T} v=\mathcal{T} \mathcal{B} v$ that is $\mathcal{B} z=\mathcal{T} z$.

Using $(d)$ again, we get

$$
d(\mathcal{A} z, \mathcal{B} z) \leq k \max \{d(\mathcal{S} z, \mathcal{T} z), d(\mathcal{A} z, \mathcal{S} z), d(\mathcal{B} z, \mathcal{T} z), d(\mathcal{S} z, \mathcal{B} z), d(\mathcal{A} z, \mathcal{T} z)\}
$$

that is

$$
d(z, \mathcal{B} z) \leq k d(z, \mathcal{B} z)
$$

Consequently, we have $z=\mathcal{B} z=\mathcal{T} z$. Hence, $\mathcal{A} z=\mathcal{S} z=z=\mathcal{B} z=\mathcal{T} z$, and so $z$ is a common fixed point of the four mappings.

Finally, we prove that $z$ is unique. Suppose that $w$ is a second distinct common fixed point of $\mathcal{A}, \mathcal{B}, \mathcal{S}$ and $\mathcal{T}$. Then, using inequality $(d)$, we obtain

$$
d(\mathcal{A} z, \mathcal{B} w) \leq k \max \{d(\mathcal{S} z, \mathcal{T} w), d(\mathcal{A} z, \mathcal{S} z), d(\mathcal{B} w, \mathcal{T} w), d(\mathcal{S} z, \mathcal{B} w), d(\mathcal{A} z, \mathcal{T} w)\}
$$
i.e.,

$$
d(z, w) \leq k d(z, w)
$$

Therefore, $z=w$.

Similarly, one can obtain this conclusion by supposing $\mathcal{A}(\mathcal{X}), \mathcal{S}(\mathcal{X})$ or $\mathcal{T}(\mathcal{X})$ is closed.

For compatibility of type $(C)$, we use the same proof and condition (3) of Proposition 2.1. 


\section{REFERENCES}

[1] H. Bouhadjera, General common fixed point theorems for compatible mappings of type $(C)$, Sarajevo J. Math., 1(14)(2005), 261-270.

[2] J. Jachymski, Common fixed point theorems for some families of mappings, Indian J. Pure Appl. Math, 25(1994), 925-937.

[3] K. Jha, R.P. Pant and S.L. Singh, Common fixed points for compatible mappings in metric spaces, Radovi Mat., 12(2003), 107-114.

[4] G. Jungck, Compatible mappings and common fixed points, Internat. J. Math. Math. Sci., 9(1986), 771-779.

[5] G. Jungck, Common fixed points for non-continuous nonself mappings on a nonnumeric spaces, Far East J. Math. Sci., 4(2)(1996), 199-212.

[6] G. Jungck, P.P. Murthy and Y.J. Cho, Compatible mappings of type (A) and common fixed points, Math. Japon., 36(1993), 381-390.

[7] H.K. Pathak, Y.J. Cho, S.M. Kang and B.S. Lee, Fixed point theorems for compatible mappings of type $(P)$ and applications to dynamic programming, Le Matematiche, (Fasc. I)(50)(1995), 15-30.

[8] H.K. Pathak, Y.J. Cho, S.M. Kang, B. Madharia, Compatible mappings of type $(C)$ and common fixed point theorems of Greguš type, Demonstratio Mathematica, XXXI(3)(1998), $499-518$.

[9] H.K. Pathak and M.S. Khan, Compatible mappings of type $(B)$ and common fixed point theorems of Greguš type, Czechoslovak Math. J., 45(120)(1995), 685-698.

\section{H. BOUHADJERA}

DÉPARTEMENT De MathÉmatiques

FACUlté DEs SCIENCES

Université BADJi MoKhtar B.P. 12

23000 ANNABA

ALGERIE

E-mail address: b_hakima2000@yahoo.fr

BRIAN FISHER

Department of Mathematics

LEICESTER UNIVERSITY

LEICESTER, LE1 7RH

ENGLAND

E-mail address: fbr@le.ac.uk 